\title{
Confined modes in finite-size photonic crystals
}

\author{
Tao Xu, Suxia Yang, Selvakumar V. Nair, and H. E. Ruda \\ Electronic and Photonic Materials Group, University of Toronto, Toronto, Canada M5S 3E4 \\ (Received 6 December 2004; revised manuscript received 10 May 2005; published 18 July 2005)
}

\begin{abstract}
The real band structure of finite-size photonic crystals (PCs) is different from the band structure of infinite PCs derived using Bloch's theorem. There are isolated instead of a continuum of modes around a band edge. We use the envelope function approximation (EFA) method to derive simple formulas to describe the frequency and field pattern of the modes in finite-size PCs. The results are compared with those from finitedifference time-domain (FDTD) method. We observe that the agreement between the results from EFA with a vanishing boundary condition and FDTD is sensitive to the boundary orientation. In addition, although a simple group-velocity-based analysis explains the long lifetime of the confined modes, this explanation is inappropriate for certain structures. In particular, we find boundary orientations, for which the lifetime of the confined modes can be enhanced by orders of magnitude compared with that anticipated from group velocity analysis. We explain this by total internal reflection. Our results demonstrate the utility of the EFA approach in studying the modes in finite-size PCs and reveal the importance of boundary conditions to develop an EFAbased tool for cavity lasers and for studying nonlinear interactions.
\end{abstract}

DOI: 10.1103/PhysRevB.72.045126 PACS number(s): 42.70.Qs, 42.25.-p, 42.55.Sa, 03.50.De

\section{INTRODUCTION}

Photonic crystals (PCs) are artificial structures with a periodic dielectric contrast. ${ }^{1,2}$ Bloch's theorem may be applied to Maxwell's equations in an infinite PC to yield the corresponding band structure. ${ }^{3-5}$ However, for devices that are not infinitely periodic compared with the wavelength of the optical states such as band edge cavities, ${ }^{6-10}$ Bloch's theorem is inapplicable since light leaks out of the structure and the discrete translational symmetry breaks down in the vicinity of the PC boundary. In this paper, we present a systematic study of the photonic states in finite-size PCs.

We focus on the frequencies around a band edge since the optical properties of a finite-size PC in this frequency range deviate most from those infinite PC counterpart and are expected to find diverse applications. Around band edges, the group velocity of propagating states approaches zero, which increases the interaction between photons and the matter system. ${ }^{11-14}$ Both optical pumped and electrically driven band edge cavity lasers have been fabricated. ${ }^{6-10}$ Nonlinear optical processes can be enhanced due to the small group velocity of light pulses. ${ }^{15-17}$ Our study should be able to make a contribution to further developments, especially physical understanding in these fields.

To make the description more transparent and the calculation more computationally time efficient, we restrict ourselves to studies of two-dimensional (2-D) PCs in this paper. However, the same concepts may readily be extended to three-dimensional (3-D) cases. The PCs considered in the calculation are comprised of a square lattice of dielectric rods in air, as illustrated in Fig. 1. Figures 1(a) and 1(b) correspond to two finite-size structures having the same structural parameters but different boundary orientations. We refer to the structure shown in Fig. 1(a), having its boundary orientated along $\Gamma-X$ direction, as the $X$ structure and that of Fig. 1(b) as the $M$ structure. The number of rods at each boundary is used to define the size of each structure. The spatial length in the structure is normalized as $\left(x^{\prime}, y^{\prime}\right)$ $=(x, y) / a$, where $a$ is the lattice constant. The band diagram for transverse magnetic (TM) modes in the corresponding infinite PC is given in Fig. 2. The calculation and interpretation of this band structure is discussed in Ref. 5 .

One salient difference we find is that there are isolated instead of a continuum of modes at a band edge. This phenomenon can be explained using a self-interference picture. The light incident internally at the interface between a PC and air will be reflected back and interfere with itself. Only those modes that satisfy the condition of constructive interference survive. In addition, our studies show that not only the amplitude of group velocities affects the cavity leakage rate, the wave vector anisotropy at a band edge and the boundary orientation also play an important role in determining the cavity leakage rate, which is unique for 2-D and 3-D PCs. Under certain conditions, an effective total internal reflection (TIR) can occur and the confined modes then have a very low corresponding leakage rate, which may find appli-

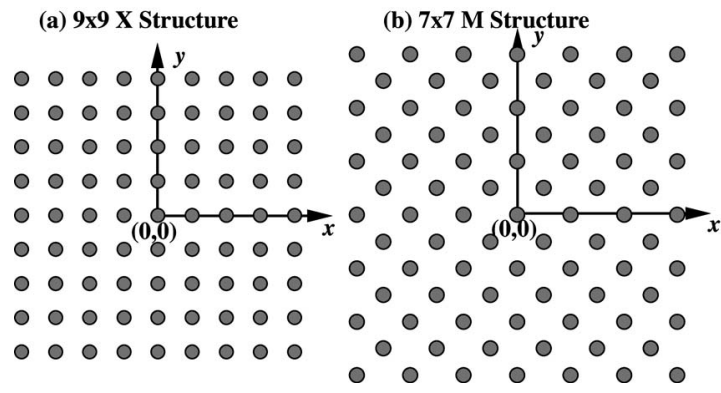

FIG. 1. Square lattice of dielectric rods in air. The dielectric constant of the rods, $\epsilon$, is 9.0 and the diameter of the rods $d=0.4 a$, where $a$ is the lattice constant of the structure. The size of the structures are defined in terms of the number of rods lying along the boundary: (a) $9 \times 9 X$ structure and (b) $7 \times 7 M$ structure. The spatial coordinate axes are illustrated in the figure, where the origin is located at the center and the length is normalized as $\left(x^{\prime}, y^{\prime}\right)$ $=(x, y) / a$. 


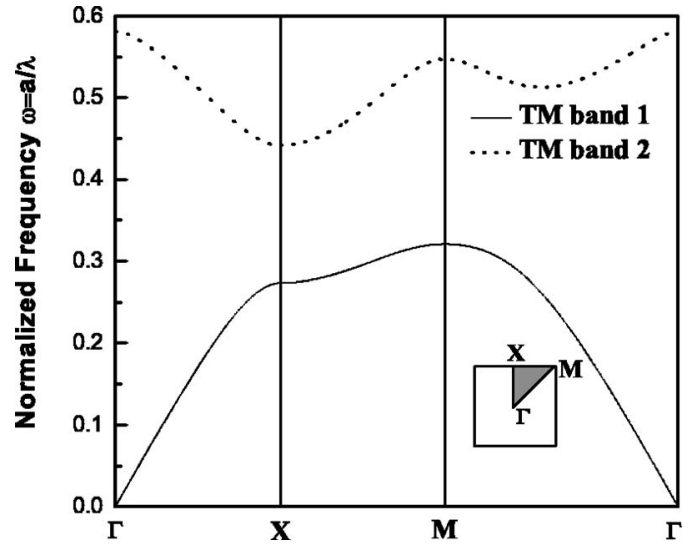

FIG. 2. The first two TM bands for the infinite size PC shown in Fig. 1. Frequencies are normalized by $\omega=a / \lambda$, where $\lambda$ is the wavelength of light in vacuum. The insert shows the first Brillouin zone in the $k$ space. TM modes are the modes having the electric field pointing out along the axis of the rods.

cations in the high- $Q$ cavity for lasers and other optical components.

The resonant modes in finite-size PCs at a band edge are well confined, especially in geometries where an effective TIR exists. This situation is analogous to that of an electron confined in an infinite potential well of finite width, where the electronic states are quantized. The most popular approach to the description of such quantized electronic states in semiconductor heterostructures is the envelope function approximation (EFA) - also referred to as the effective mass approximation. ${ }^{18,19}$ For the cases of finite-size PCs, the highly confined resonant modes can also be described using a similar EFA, which is more physically intuitive and time efficient compared with a numerical method such as finitedifference time-domain (FDTD). By analogy, one could term these resonant modes "quantized optical modes" in a photonic "quantum box."

The rest of the paper is organized as follows. In Sec. II, we discuss the computational methods employed. In Sec. III, we analyze the mode patterns and the corresponding frequencies of the confined modes in finite-size PCs at the first TM band edge. EFA is used to describe the behavior of the confined modes. We show that the EFA results agree strikingly well with those from FDTD calculations for the highly confined modes. We also investigate the modes for the cases of relatively weak confinement. It is demonstrated that the EFA approach is reliable, however it is sensitive to boundary orientations. In Sec. IV, one of the most important properties of the confined modes in finite-size PCs, the lifetime, is studied in detail. The relationship between the leakage rate and the boundary orientation and size of the structure is discussed. We argue that the leakage rate can be decreased greatly through effective TIR, where light in the finite-size PCs cannot couple out into the modes in air owing to a mismatch of the wave vectors. The small leakage in the case of effective TIR also confirms our assumption regarding the boundary conditions for EFA in Sec. III and reveals the importance of boundary conditions to develop an EFA-based tool for cavity analysis.

\section{COMPUTATIONAL METHOD}

The band diagram in Fig. 2 was computed using the MIT Photonic Bands (MPB) software,$^{20}$ which is based on a plane wave expansion (PWE) method. In the following section, we will use this method for infinite PCs to describe the properties of photonic states, which include the group velocity and dispersion relation around band edge. To calculate the optical properties of confined modes in finite-size PCs, a 2-D FDTD method based on Yee's algorithm ${ }^{21}$ is used, which provides accurate numerical solution to 2-D Maxwell's equations. The FDTD algorithm is widely used to characterize optical microcavities. ${ }^{22-26}$ For a review of current practice in FDTD simulations, see Ref. 27. A perfectly matched layer (PML) boundary condition is used to absorb outgoing waves without any spurious reflection. ${ }^{28}$ Throughout the paper the frequency $\omega$ is normalized to be $\omega=a / \lambda$ as shown in the band structure Fig. 2, where $\lambda$ is the wavelength of light in vacuum.

To make the calculation results accurate and the comparison between the PWE method and FDTD method reasonable, we have to make the two computational methods converge. We adopt the spatial grid size as $1 / 64 a$ in the PWE calculation, which we have verified to have converged by comparing with the results for $1 / 128 a$ grid size. For the FDTD calculations we adopt the grid size as $\Delta x=\Delta y$ $=1 / 64 a$, which is comparable to that used in the PWE and small enough to resolve the features in the field and structure for the frequency region we studied. To make the computation stable, ${ }^{27}$ the time step we used is $\Delta t=\frac{1}{2}(\Delta x / c)$ $=\frac{1}{128}(a / c)$, where $c$ is the light speed in vacuum.

A pointwise source is introduced to excite the modes, which is more flexible than plane waves since its $k$ vectors are two-dimensional. ${ }^{24,27}$ To find the confined modes in finite-size PCs, we excite it with an impulse, which has a broad spectrum. ${ }^{29}$ We record the electrical field for TM modes at different spatial locations in the structure for an extended period (normally $2^{19} \times \Delta t$ ) and transform the time response to a spectral response using Fourier analysis. The longer the time period we record for, the better the resolution of the spectral response. From the spectrum, we can select the frequency peaks, which are the confined modes in the structure. Once we have these frequencies, we can determine the corresponding spatial field distribution by performing a second FDTD calculation and extract the spatial field pattern that evolves coherently in time with a well-defined single frequency centered at the peak frequency. The basic procedure is analogous to the Correlation Method used by the propagating beam method (PBM) to determine wave guide modes. ${ }^{30}$ The second FDTD run has the same time length as the first one in our case.

We use the quality factor $(Q)$ to evaluate the lifetime of the confined modes. Normally there are two ways to determine the $Q$ value for a certain mode in an optical cavity. First, the $Q$ value can be estimated using the frequency spectrum from $Q=\omega_{0} / \Delta \omega$, where $\omega_{0}$ is the central frequency of the modes and $\Delta \omega$ is the full width at half maximum (FWHM) of the frequency peak. Second, since the energy in the microcavity decays as $\exp ^{\left(-\omega_{0} t / Q\right)}$, we can estimate $Q$ 
from the time response. The second approach is selected since it is more accurate than the first for high- $Q$ modes as the frequency resolution is limited by the length of time data. In the FDTD simulation, we use a Gaussian pulse whose envelope function is given by $\exp ^{-(t / T)^{2}}$ to excite the structure, where $T$ is defined as pulse time. Since the energy decays as $\exp ^{\left(-\omega_{0} t / Q\right)}$, the field amplitude decays as $\exp ^{\left(-\omega_{0} t / 2 Q\right)}$. We monitor the field envelope time response at a number of points in the structure. If the Gaussian pulse source excites only one mode, the envelope time response is

$$
\begin{aligned}
g(t) & =\int_{0}^{t} \exp ^{-(\tau / T)^{2}} \times \exp ^{\left(-\omega_{0} / 2 Q\right)(t-\tau)} d \tau \\
& =\left[\frac{\sqrt{\pi} T}{2} \exp ^{\left(T / 2 T_{\text {dacay }}\right)^{2}} \operatorname{erf}\left(\frac{t}{T}-\frac{T}{2 T_{\text {decay }}}\right)\right] \exp ^{-\left(t / T_{\text {decay }}\right)},
\end{aligned}
$$

where the decay time $T_{\text {decay }}$ is defined as $T_{\text {decay }} \equiv 2 Q / \omega_{0}$. It is clear that for highly confined modes where $T_{\text {decay }} \gg T$, for $t$ $\gtrsim 4 T$ the decay behavior of the envelope is dominated by $\exp ^{\left(-t / T_{\text {decay }}\right)}$ since $\operatorname{erf}\left(t / T-T / 2 T_{\text {decay }}\right) \rightarrow 1$ when $t \gtrsim 4 T$. When we obtain the field envelope response at a particular spatial location in the structure and extract the decay time $T_{\text {decay }}$ after the excitation Gaussian pulse has died off, the $Q$ value can be determined from the decay time and the frequency using $Q=\frac{1}{2} T_{\text {decay }} \omega_{0}$. All $Q$ values reported below were obtained using this method., ${ }^{5,24}$ To make sure the Gaussian pulse only excites one mode, its pulse time $T$ must be long enough to have a narrow frequency spectrum, which only covers the frequency peak with which we are concerned. The typical pulse time in our cases is in the range of $T$ $=2^{15}-2^{17} \times \Delta t$.

\section{QUANTIZATION OF CONFINED MODES}

In this section, we concentrate on the first band edge for TM modes to illustrate the quantization. For the confined modes in this frequency range, the leakage rate for $X$ structures is much less than for $M$ structures. In $X$ structures, there is an effective total internal reflection (TIR) while in $M$ structures, the light impinges almost perpendicularly to the boundary and leaks out much faster. This point will be discussed in detail in the next section. As a result, we may consider $X$ structures as a "closed" system where little leakage is present and $M$ structures as a partially "open" system, analogous to a Fabry-Perot cavity.

In infinite PCs defined by the periodic dielectric constant $\epsilon(\mathbf{r})$, the electrical field modes $\mathbf{E}_{n \mathbf{k}}$ of the TM mode satisfy the wave equation

$$
\boldsymbol{\nabla} \times\left[\boldsymbol{\nabla} \times \mathbf{E}_{n \mathbf{k}}(\mathbf{r})\right]=\frac{\omega_{n}^{2}}{c^{2}} \epsilon(\mathbf{r}) \mathbf{E}_{n \mathbf{k}} .
$$

By the Bloch-Floquet theorem, $\mathbf{E}_{n \mathbf{k}}$ takes the form

$$
\mathbf{E}_{n \mathbf{k}}(\mathbf{r})=\mathbf{u}_{n \mathbf{k}} e^{i \mathbf{k} \cdot \mathbf{r}},
$$

where the Bloch functions $\mathbf{u}_{n \mathbf{k}}$ have the periodicity of the lattice. ${ }^{4,5}$
In our calculation, the size of the structure is about 10-20 times the lattice constant $a$. We may assume the confinement as a perturbation of the periodic "potential" and the confined mode as a perturbation of the Bloch mode for infinite PCs. The electrical field of the perturbed mode in finite-size PCs may be expressed as

$$
\mathbf{E}_{\lambda}(\mathbf{r})=\sum_{n} \int W_{n}(\mathbf{k}) \mathbf{E}_{n \mathbf{k}} d \mathbf{k},
$$

where $W_{n}(\mathbf{k})$ are the expansion functions of the mode in $\mathbf{k}$ space and the subscript $\lambda$ is used to denote the frequency and the field of the mode in the finite-size PCs. Since, in our study, we have a nondegenerate band and there is a band gap separating the first TM band from other bands as illustrated in Fig. 2, we may neglect the coupling between bands and only expand the perturbed mode in the first TM band:

$$
\mathbf{E}_{\lambda}(\mathbf{r})=\int W_{1}(\mathbf{k}) \mathbf{E}_{1 \mathbf{k}} d \mathbf{k} .
$$

There are a number of theoretical formulations of the EFA method as applied to the photonic crystal heterostructures. ${ }^{31-33}$ In Ref. 31, it is deduced that if we define envelope function $F_{1}(\mathbf{r})$ as

$$
F_{1}(\mathbf{r})=\int W_{1}(\mathbf{k}) e^{i \mathbf{k} \cdot \mathbf{r}} d \mathbf{k},
$$

it will follow the envelope equation

$$
\omega_{1}^{2}(-i \boldsymbol{\nabla}) F_{1}(\mathbf{r})=\omega_{\lambda}^{2} F_{n}(\mathbf{r}),
$$

where $\omega_{1}^{2}(-i \boldsymbol{\nabla})$ is the operator obtained from $\omega_{1}^{2}(\mathbf{k})$ by replacing the wave vector components $k_{x}, k_{y}$, with the derivative $-i \partial / \partial x,-i \partial / \partial y$.

In our calculation, we may expand the operator $\omega_{n}^{2}(-i \boldsymbol{\nabla})$ in the vicinity of the wave vector $\mathbf{k}_{M}$. Since it has been demonstrated that, at the band edge, parabolic approximation is applicable,$^{34}$ we may write the operator as

$$
\omega_{1}^{2}(-i \boldsymbol{\nabla})=\omega_{1}^{2}\left(\mathbf{k}_{M}\right)+b\left[\left(\frac{1}{i} \frac{\partial}{\partial x}-k_{M x}\right)^{2}+\left(\frac{1}{i} \frac{\partial}{\partial y}-k_{M y}\right)^{2}\right],
$$

where $b=\left.\left(\partial^{2} \omega_{1}^{2} / \partial k_{x}^{2}\right)\right|_{\mathbf{k}_{M}}=\left.\left(\partial^{2} \omega_{1}^{2} / \partial k_{y}^{2}\right)\right|_{\mathbf{k}_{M}}$ describes the dispersion at $\mathbf{k}=\mathbf{k}_{M}$.

This suggests the following solution for the envelope equation,

$$
F_{1}(\mathbf{r})=e^{i \mathbf{k}_{M} \cdot \mathbf{r}} f_{1}(\mathbf{r}),
$$

where the function $f_{1}(\mathbf{r})$ satisfies the equation:

$$
-b\left(\frac{\partial^{2}}{\partial x^{2}}+\frac{\partial^{2}}{\partial y^{2}}\right) f(\mathbf{r})=\left[\omega_{\lambda}^{2}-\omega_{1}^{2}\left(\mathbf{k}_{M}\right)\right] f(\mathbf{r}) .
$$

Following the approach in Ref. 31, the electrical field of the confined modes in our finite-size PCs may be written

$$
\mathbf{E}_{\lambda}(\mathbf{r})=F_{1}(\mathbf{r}) u_{1, \mathbf{k}_{M}}=f_{1}(\mathbf{r}) \mathbf{E}_{1 \mathbf{k}_{M}}(\mathbf{r}) .
$$

As discussed above, the envelope function approximation (EFA) provides a simple description of the confined modes. 
On the length scale of the lattice constant, the field retains features similar to that of the bulk material, but its envelope is defined by the envelope function, whose feature length is comparable to the characteristic length of finite-size PCs.

We now solve Eq. (10) for the modes in the structures as illustrated in Fig. 1. In all cases we consider, air surrounds finite-size PCs. At the interface between the finite-size PC and air, the envelope function satisfies boundary conditions. As we show later, for the cases of $X$ structures, owing to TIR, $Q$ values of the confined modes are very high. We can assume that the effective confining "potential" is infinite and that the envelope function vanishes at the boundary of the finite-size PCs. In Ref. 35, it is justified that the correct boundary condition at an interface with an impenetrable barrier is that the envelope function should be zero there for the EFA used in electronic crystals, which is similar to our TIR cases here. If we use a box to replace the finite-size PCs with boundaries at $x_{1}=0, x_{2}=L, y_{1}=0, y_{2}=L$ and assume that the envelope function vanishes at the boundaries of the box, then Eq. (10) can be solved exactly and gives

$$
\begin{gathered}
f(x, y)=\sin \left(K_{x} x\right) \sin \left(K_{y} y\right), \\
K_{x} L=m \pi, \quad K_{y} L=n \pi,
\end{gathered}
$$

where $m, n$ are positive integers.

The corresponding frequencies of the confined modes are

$$
\omega_{\lambda}^{2}=\omega_{1}^{2}\left(\mathbf{k}_{M}\right)+b \frac{\pi^{2}}{L^{2}}\left(m^{2}+n^{2}\right) .
$$

In $M$ structures, one cannot assume that the envelope function vanishes at the boundaries since the light in these structures is incident perpendicular to the boundary and there is no TIR. The boundary conditions for two media with different crystal structure remains a subject of contention ${ }^{36}$ and requires further study. We will not deal with this question in this paper. We assume the envelope function vanishes at the boundary for $M$ structures, too, and test the validity of this assumption using the FDTD calculations. With such an assumption, the mode profile and frequency position of confined modes in the $M$ structure can be described similarly to that of $X$ structures.

In the following discussion, the FDTD method is used to check the applicability of EFA and gives the properties of the confined modes. First, we investigate the confined modes in $X$ structures. We choose a $13 \times 13 X$ structure as an example. To obtain the frequencies of the confined modes, a pointwise source is introduced in the top right corner [corresponding to the coordinate $(6,6)$ in Fig. 1(a)] of the structure. Since this pointwise source is placed at a low symmetry point and not at the nodes of any modes, all modes (except some degenerate modes) in the structure can be excited. Time monitors are placed at the center of the rods along the $\Gamma-M$ and $\Gamma-X$ directions to record the field response at the spatial points they are placed. In the spectrum shown in Fig. 3, there are several isolated peaks around the band edge, which confirms our supposition that there is no continuum of photonic states for finite-size PCs.
We can extract the mode profile of these confined modes at the frequency peak in the spectrum using the FDTD method. It is found that the mode pattern from FDTD is consistent with that obtained by EFA. The mode patterns clearly appear as the band-edge Bloch function that oscillates on the lattice length scale modulated by the envelope functions on the length scale of the structure. The envelope of the extracted mode profile agrees well with the envelope function given by Eq. (12a). The modes are identified using "quantum numbers" $m, n$ in Eq. (12b). The six peaks of Fig. 3 correspond to modes $(1,1),(1,2),(2,2),(1,3),(2,3)$, and $(1,4)$, respectively. The asymmetric modes such as $(1,2),(1$, $3),(2,3)$, and $(1,4)$ are degenerate modes and any linear combination of two such degenerate modes is still a cavity mode corresponding to the same frequency. When a point source is placed at the upper right corner $(6,6)$, the mode $[(1,3)+(3,1)]$ is excited. If the point source is placed at the center of the boundary $(0,6)$, the mode $[(1,3)-(3,1)]$ is excited.

To compare the FDTD and EFA, we plot the mode profiles of the fundamental mode, mode $(2,2)$, and two degenerate modes, $[(1,3)+(3,1)]$ and $[(1,3)-(3,1)]$ in Fig. 4 . The pictures on the left are obtained from FDTD calculations and the pictures on the right are given from Eq. (12a). It is clear that the mode patterns given by the two methods look very similar.

To study this in more detail, we take a cross section of the mode patterns from the FDTD calculations along a certain line and compare it with an envelope function along the same line as predicted by EFA. As illustrated in Fig. 5, the envelope functions from EFA match accurately with the envelope of the modes from the FDTD calculation, which confirms the applicability of EFA to estimate the mode field patterns in $X$ structures.

In addition to the mode pattern, EFA also predicts the frequency positions of the modes as given by Eq. (13). To verify this equation, we determine $b=\left.\left(\partial^{2} \omega^{2} / \partial k_{x}^{2}\right)\right|_{\mathbf{k}_{M}}$ by fitting the dispersion curve calculated using the PWE method in the frequency range of the modes. In Fig. 6, we compare the frequency positions obtained from FDTD calculations and those predicted by the EFA method. The assumption used in Eq. (13) to calculate the frequency position of the modes is that the dispersion surface at the band edge is isotropic and parabolic. However, away from the band edge, this assumption is not valid as shown in the Appendix. We have to modify the dispersion relation by adding higherorder terms. In our case, the lowest-order correction to the parabolic dispersion is fourth order in $\mathbf{k}$. As shown in the Appendix, the modified dispersion equation describes the real situation more accurately for modes further away from the band edge. The EFA equation for the envelope function of higher-order modes becomes complicated with the presence of the fourth-order differential operators. However, a vanishing boundary condition simplifies the problem as the solution to the envelope equation with a parabolic dispersion relation is still a solution to the new modified envelope equation with the fourth-order terms. A similar behavior has been noted for the electronic structure of quantum dots by comparison of tightly binding and effective mass approximation 


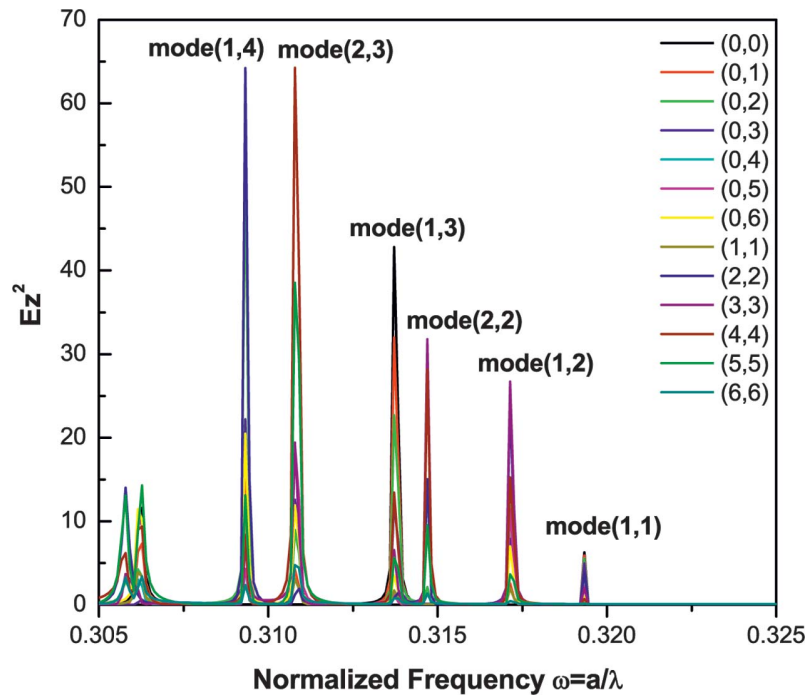

FIG. 3. (Color) The spectrum obtained for a $13 \times 13 X$ structure at the first TM band edge. Data is gathered from point time monitors placed at different positions and then collected together. There are 13 time monitors in the structure, whose coordinates are displayed in the legend.

methods. ${ }^{37}$ This explains why the mode patterns expected by EFA with parabolic dispersion relation agree well with real patterns calculated from FDTD even for the high-order $(1,4)$ mode, as shown in Fig. 5. However, the frequency positions expected using the new envelope equation are different. The modified equation is

$$
\omega_{\lambda}^{2}=\omega_{1}^{2}\left(\mathbf{k}_{M}\right)+b \frac{\pi^{2}}{L^{2}}\left(m^{2}+n^{2}\right)+c \frac{\pi^{4}}{L^{4}}\left(m^{4}+n^{4}\right)+d \frac{\pi^{4}}{L^{4}} m^{2} n^{2},
$$

where the parameters $b=\left.\left(\partial^{2} \omega_{1}^{2} / \partial k_{x}^{2}\right)\right|_{\mathbf{k}_{M}}, \quad c=\left.\left(\partial^{4} \omega_{1}^{2} / \partial k_{x}^{4}\right)\right|_{\mathbf{k}_{M}}$, and $d=\left.\left(\partial^{4} \omega_{1}^{2} / \partial k_{x}^{2} \partial k_{y}^{2}\right)\right|_{\mathbf{k}_{M}}$ can be obtained by fitting the dispersion surface at the band edge as described in the Appendix.

In Fig. 6(a), the frequency positions from the FDTD calculations are shown, together with the expected values from a parabolic fit of Eq. (13) and the fourth-order fit of Eq. (14) for the confined modes in a $13 \times 13 X$ structure. Another way to predict the frequency positions with EFA is to calculate the corresponding frequency position of the mode $\left(k_{M x}\right.$ $\left.-m \pi / L, k_{M y}-n \pi / L\right)$ with the PWE method, which provides the dispersion surface at the band edge accurately to all orders. From the figure, it can be seen that the parabolic fit does not follow the trend of the data from the FDTD, especially for asymmetric modes such as $(1,3)$ and $(1,4)$. The fourth-order fit agrees well with the fit from the PWE, which confirms that the fourth-order dispersion equation describes the real dispersion surface at the band edge in this frequency range. The fourth-order fit also follows the trend of FDTD results. The relative error 1 defined as $\mid\left(\omega_{\mathrm{FDTD}}\right.$ $\left.-\omega_{\text {fourthorder }}\right) /\left(\omega_{1, \mathbf{k}_{M}}-\omega_{\mathrm{FDTD}}\right) \mid$ is in the range of $11.1 \%$ to $12.8 \%$ for all six modes. This discrepancy between the FDTD data and the EFA predictions can partially be explained by the assumption of a vanishing boundary condi-

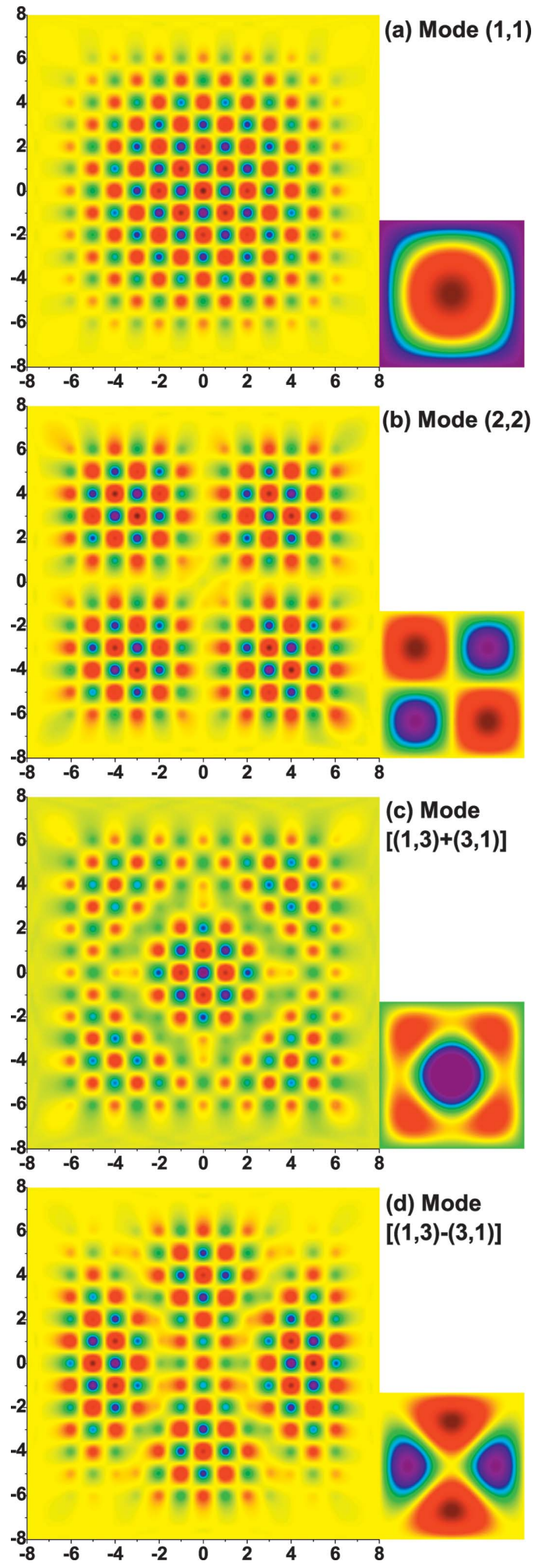

FIG. 4. (Color) The mode profiles of modes (a) $(1,1)$, (b) $(2,2)$, (c) $[(1,3)+(3,1)]$, and (d) $[(1,3)-(3,1)]$. The pictures on the left are obtained using the FDTD method and the pictures on the right are given using Eq. (12a).

tion. At the assumed boundaries of the structure, the envelope of the data computed from FDTD still has a finite value instead of zero as shown in Fig. 5. With an increase in size, 
(a) Mode $(1,1)$ along $\Gamma-M$

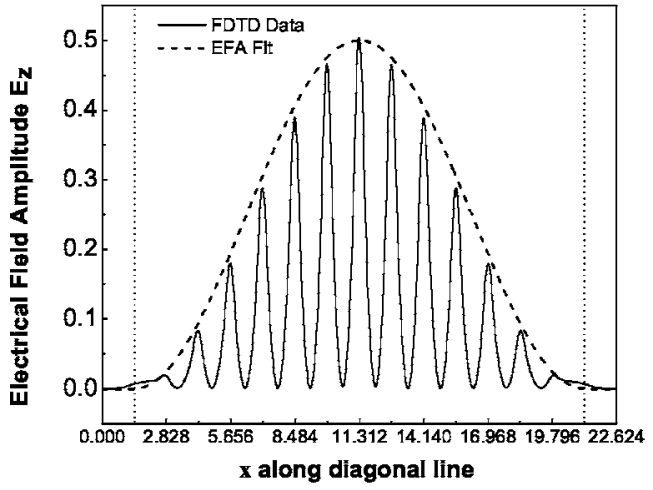

(c) Mode $[(1,3)+(3,1)]$ along $\Gamma-M$

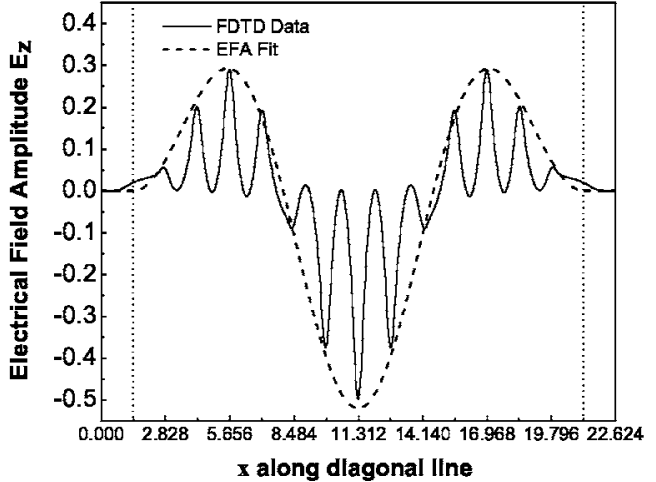

(b) Mode $(1,1)$ along $\Gamma-X$

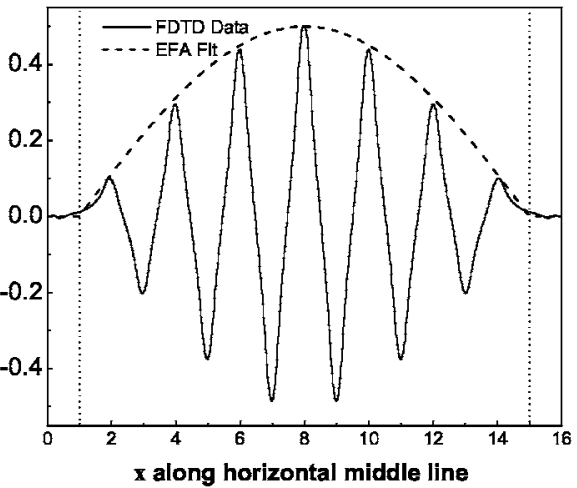

(d) Mode $[(4,1)-(1,4)]$ along $\Gamma-M$

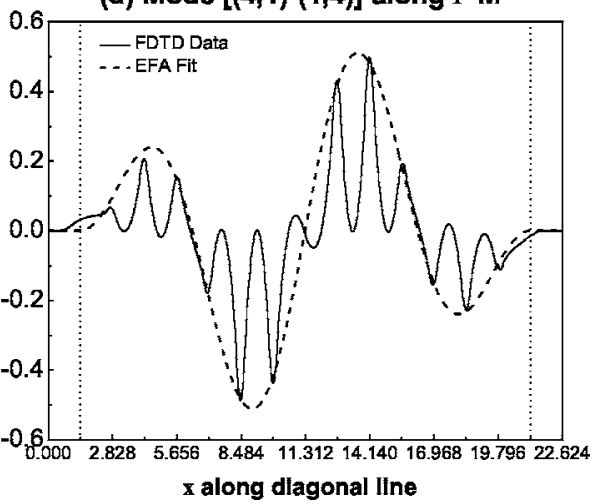

FIG. 5. Cross sections of the mode patterns for a $13 \times 13 X$ structure (a) along the diagonal line for mode $(1,1)$, (b) along the horizontal middle line for mode $(1,1),(\mathrm{c})$ along the diagonal line for mode $[(1,3)+(3,1)]$, and $(d)$ along the diagonal line for mode $[(4,1)-(1,4)]$ from point $(-6,6)$ to point $(6,-6)$. Solid black lines are extracted from the mode pattern obtained using the FDTD method. Dashed lines are predicted from Eq. (12a), where the length $L$ is assumed to be $n a$, with $n$ the number of the rods along the boundary. Vertical dotted lines indicate the assumed boundaries of the EFA box. The unit in the plots is $a$, the lattice constant. the confinement of the modes in $X$ structures becomes much better as we will discuss in the following section. Thus it appears that a vanishing boundary condition is more applicable in bigger structures and the relative error between FDTD and EFA decreases with increase in the size. The frequency position of the fundamental mode for the $X$ structure is shown as a function of size in Fig. 6(b). It can be seen that the relative error 1 decreases to below $4 \%$ when the size increases to 19 , in agreement with the above argument. However, when the size increases to 21 , there is fluctuation of the relative error 1 , which increases to $6.5 \%$ instead of decreasing. We regard this phenomenon as the discrepancy between the FDTD calculation and the PWE calculation. The band edge frequency $\omega\left(\mathbf{k}_{M}\right)$ obtained from the PWE calculation is not necessarily in agreement with the band edge frequency in the FDTD calculations. The little shift of band edge frequency will bring big fluctuation of relative error 1 since the frequency of the fundamental modes is quite near to the band edge frequency. To eliminate the influence of the band edge frequency, we define another relative error 2 as $\mid\left[\omega(1,1)^{2}\right.$ $\left.-\omega(1,3)^{2}\right]_{\mathrm{FDTD}}-\left[\omega(1,1)^{2}-\omega(1,3)^{2}\right]_{\text {fourthorder }} / /\left[\omega(1,1)^{2}\right.$

$\left.-\omega(1,3)^{2}\right]_{\text {FDTD }}$. From Eq. (14), it is clear that the relative error 2 has no dependency of the band edge frequency. Shown in Fig. 6(b), relative error 2 almost monotonously decreases with the increase of size. (a) Modes in a $13 \times 13 \times$ Structure

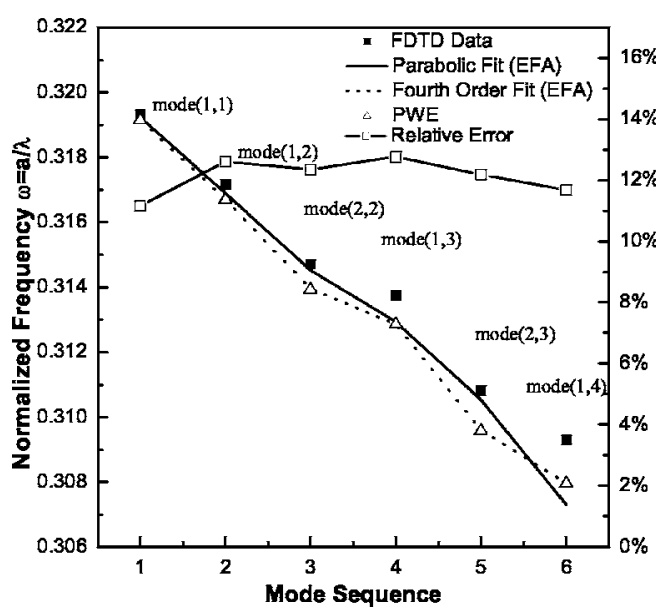

(b) Size-dependence of the frequency of the fundamental mode

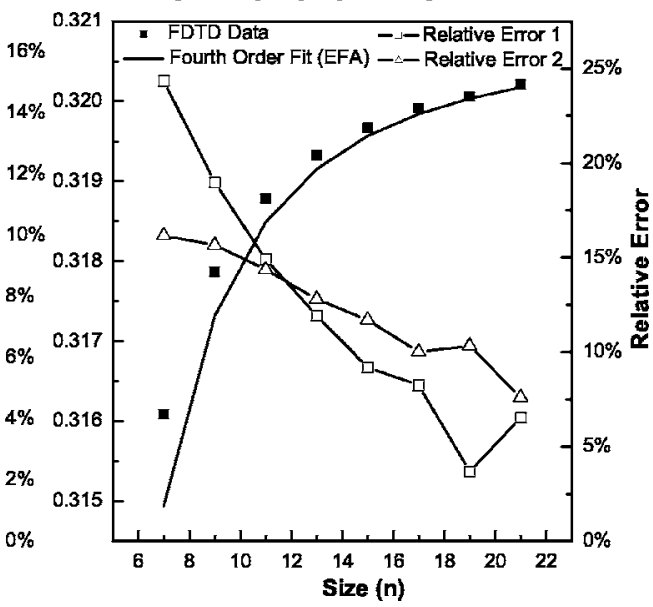

FIG. 6. Comparison between FDTD data and expected values from EFA. (a) The frequency of the confined modes in a $13 \times 13 X$ structure. (b) The size dependence of the frequency of the fundamental mode in the $X$ structure. The parameters of the fourth-order fit are $\omega_{1}\left(\mathbf{k}_{\mathbf{M}}\right)$ $=0.3208, \quad b=-0.08816\left(1 / \pi^{2}\right)$, $c=0.1225\left(1 / \pi^{4}\right), \quad$ and $\quad d=$ $-0.5483\left(1 / \pi^{4}\right) \quad$ (see text for details). 
(a) Mode $(1,1)$ along $\Gamma-M$

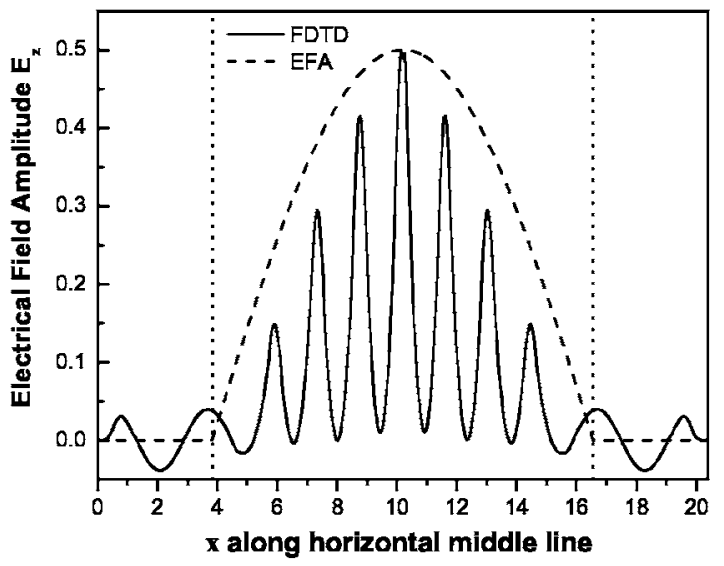

(b) Size-dependence of the frequency of the fundamental mode

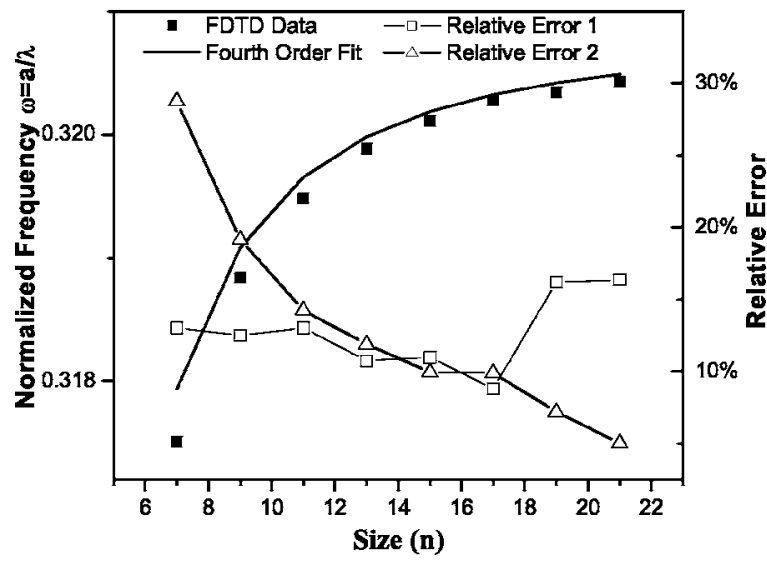

FIG. 7. (a) The cross section of the mode pattern along the horizonal middle line for the fundamental mode in a $9 \times 9 \mathrm{M}$ structure. (b) The size dependence of the frequency of the fundamental mode in $M$ structures.

We now investigate the confined modes in $M$ structures. The situation is more complex than for $X$ structures since there is no TIR and thus a vanishing boundary condition is in fact not a good assumption.

As shown in Fig. 7(a), the agreement between EFA with a vanishing boundary condition and FDTD data is much poorer than for $X$ structures. Taking a cross section of the mode pattern along a specific line, one finds that although the curve predicted by EFA follows the envelope of the mode, there is a bigger discrepancy between them as compared with $X$ structures, especially at the assumed box boundaries. However, for the frequency positions, the agreement between FDTD and EFA is relatively good. The relative error 1 is in the range of $8 \%$ to $17 \%$ in the size range of 7 to 21 . We expect the fluctuation of the relative error 1 in the bigger size is the result of the shift of band edge frequency from PWE to FDTD. In Fig. 7(b), the relative error 2 decreases from 29\% to 5\% almost monotonously. The above data show that EFA with a vanishing boundary condition can be used to describe the confined modes in $M$ structures qualitatively. Nevertheless, to predict the optical properties quantitatively, we need to derive a more appropriate boundary condition at the inter- face between the PCs and the surrounding uniform dielectric media. This is left for future study.

\section{QUALITY FACTORS OF QUANTIZED MODES AND TOTAL INTERNAL REFLECTION}

In this section, we analyze another important property of these confined modes, the lifetime. Finite-size PCs can be viewed as a cavity and the confined modes can be viewed as cavity modes. The $\mathrm{Q}$ value is used to evaluate the lifetime of these modes with the method described in Sec. II. In PCs, the group velocity becomes very small at the photonic band edge. In some bands, the group velocity can be small over a wide spectral range, referred to as group-velocity anomalies. ${ }^{12}$ The small group velocity results in enhancement of various optical processes, such as nonlinear interaction and optical amplification ${ }^{11-13,15-17}$ and reduces the leakage of the modes in finite-size PCs.

Normally, a slab of PC of length $L$ oriented along the direction $\hat{q}$ may be modeled by regarding it as a standard Fabry-Perot resonator slab whose refractive index is replaced by an effective refractive index $n_{\text {eff }}=c /\left|\hat{q} \cdot v_{m}\right|,{ }^{13,14}$ where $v_{m}$ is the group velocity of the mode. The $Q$ value of one optical mode in a 1-D Fabry-Perot cavity is $Q_{m}=2 \pi n_{\mathrm{eff}} L / \lambda_{m} t_{m},{ }^{38}$ where $\lambda_{m}$ is the wavelength of the optical mode in air, $L$ is the cavity length, and $t_{m}$ is fractional energy loss per pass through the cavity. When light is incident perpendicular to the end face of the cavity and absorption loss is ignored, one can calculate $t_{m}$ as $\ln \left(1 / R_{\text {eff }}\right)=\ln \left[\left(n_{\text {eff }}+1\right) /\left(n_{\text {eff }}-1\right)\right]^{2}$ and the $Q$ value is given by

$$
Q_{m}=2 \pi n_{\mathrm{eff}} \frac{L}{\lambda_{m} \ln \left(\frac{n_{\mathrm{eff}}+1}{n_{\mathrm{eff}}-1}\right)^{2}} .
$$

One can calculate the group velocity $v_{m}$ from the gradient of the dispersion surface at the corresponding $\mathbf{k}$ value. In infinite PCs, the group velocity becomes zero at the band edge, whereas $\left|v_{\text {group }}\right|$ in finite-size PCs is always nonzero. However, $\left|v_{m}\right|$ will decrease as the effective length of the structure is increased since the mode frequency moves toward the band edge as seen in Figs. 6(b) and 7(b). As a result, one expects that the $Q$ value of confined modes in finite-size PCs will increase with the size of the structure.

However, the above argument is applicable only to the case of perpendicular incidence. When the incidence angle is not zero, the effective refractive index of the media and also the incidence and transmitted angles at the interface determine the transmittance. In such cases, we must investigate the equi-frequency surface (EFS) plot to determine the coupling between modes in PCs and modes in air. ${ }^{34}$ As will be demonstrated below, in structures with a special boundary orientation, "total internal reflection" (TIR) significantly lowers the leakage rate of the confined modes.

To show the dependence of the leakage rate on size and boundary orientation, the $Q$ values of the fundamental mode around the first TM band edge in $X$ and $M$ structures, using the FDTD method, are discussed. The results for $X$ and $M$ structure for various sizes are plotted in Fig. 8. For compari- 
(a) Size-dependence of $Q$ values in $X$ structure

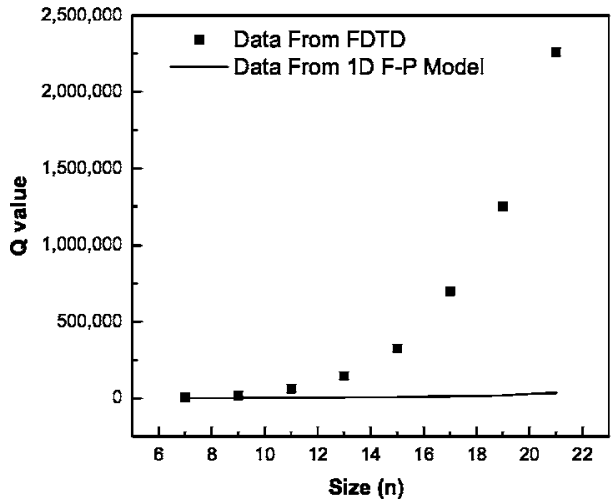

(b) Size-dependence of $Q$ values in $M$ structure

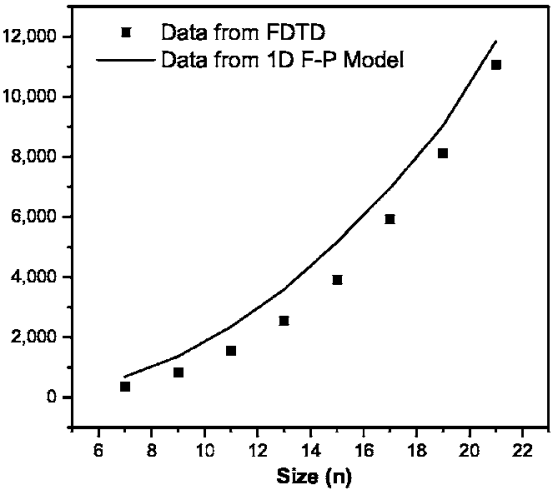

FIG. 8. (a) Size dependence of the $Q$ value of the fundamental modes in (a) $X$ structure and (b) $M$ structure. son, the $Q$ value is estimated using a 1-D Fabry-Perot model from Eq. (15). The group velocity amplitude $\left|\hat{q} \cdot v_{m}\right|$ is obtained from the gradient of the dispersion surface with respect to the $\mathbf{k}$ vector along the $\Gamma-M$ direction in the $M$ structure, and along the $X-M$ direction in the $X$ structure, at the corresponding frequency position of the fundamental modes. We take the effective length $L$ of the $X$ structure to be $n a$, and for the $M$ structure $\sqrt{2} n a$, where $n$ is the number of rods along each boundary and $a$ is the lattice constant. Figure 8 shows the $Q$ values calculated with these parameters using the 1-D Fabry-Perot model along with the values computed using the FDTD method.

From Fig. 8 it is clear that the $Q$ values increase with size as expected. However, the behavior of the mode leakage in the structures with different boundary orientation is quite different. For the $9 \times 9 X$ structure with 81 rods, the $Q$ value of the fundamental mode is 18400 , about 18 times the value calculated from the 1-D Fabry-Perot model. For the 9 $\times 9 M$ structure with 145 rods, the fundamental mode has a $Q$ value of 820 , slightly below the value of 1200 , calculated using the 1-D Fabry-Perot model. When the size increases, the increase of the $Q$ values as computed by the FDTD method agrees well with the 1-D Fabry-Perot model for $M$ structures. However, for $X$ structures, the discrepancy is huge and the $Q$ value is more than a million in the structure with 19 rods along each boundary, which is about 190 times larger than the one expected from the 1-D Fabry-Perot model. The above results tell us that in finite-size 2-D or 3-D PCs, boundary orientation is crucial to determine the coupling between states in the PCs and in air.

The confined modes near the band edge in finite-size PCs can be viewed as a mixture of photonic states around the band edge. As a result, we can investigate the coupling of confined modes in finite-size PCs and the modes in air qualitatively by examining the EFS plot obtained by the PWE. The EFS for the first TM band in the PC and the modes in air are shown in Fig. 9. Around the band edge, $\mathbf{k}$ vectors are anisotropic and point along the $\Gamma-M$ direction. We take a state at a normalized frequency of 0.32 with the wave vector pointing along $\Gamma-M$ as an example to show the coupling. The EFS of the states with normalized frequency of 0.32 in air is plotted as a circle of radius $k=0.64 \pi / a$. The coupling requires continuity of the tangential components of $\mathbf{k}$ across the interface. ${ }^{34}$ In $M$ structures with boundaries along the $\Gamma-M$ direction, the mode considered is perpendicularly in- cident to the boundary and thus it is a reasonable approximation to apply the 1-D Fabry-Perot model, as verified by data in Fig. 8(b). In $X$ structures with boundary along the $\Gamma-X$ direction, the mode considered in the PC has an incidence angle of $45^{\circ}$ and there is no coupling to light in air since the tangential component of its $\mathbf{k}$ vector exceeds the corresponding one in air. The situation is like TIR in a uniform dielectric slab with higher dielectric constant than the surrounding media with the incident angle exceeding the critical angle. In finite-size PCs, the actual modes are not so strictly confined in $\mathbf{k}$ space because of the finite size of the structures. Some photonic states with $\mathbf{k}$ whose tangential components fall in the region of the EFS in air can couple out to the states in air, which is the origin of the leakage in $X$ structures. Since most of the photonic states are still internally reflected into the structure, the $Q$ values of the confined modes in $X$ structures are much larger than those in $M$ structures, even with smaller size, which is already seen in Fig. 8. With increase in size, the confined photonic states moves

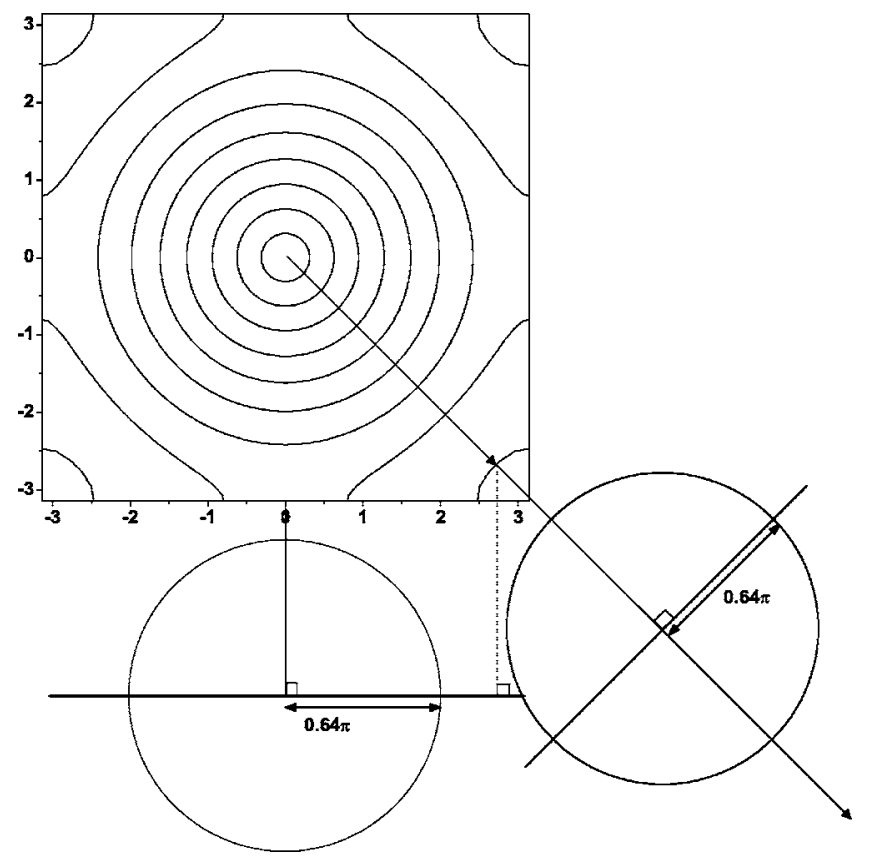

FIG. 9. The EFS of the first TM band in the infinite PC corresponding to the structures shown in Fig. 1 and the EFS of the photonic states in air. The unit of $\mathbf{k}$ is $1 / a$. 


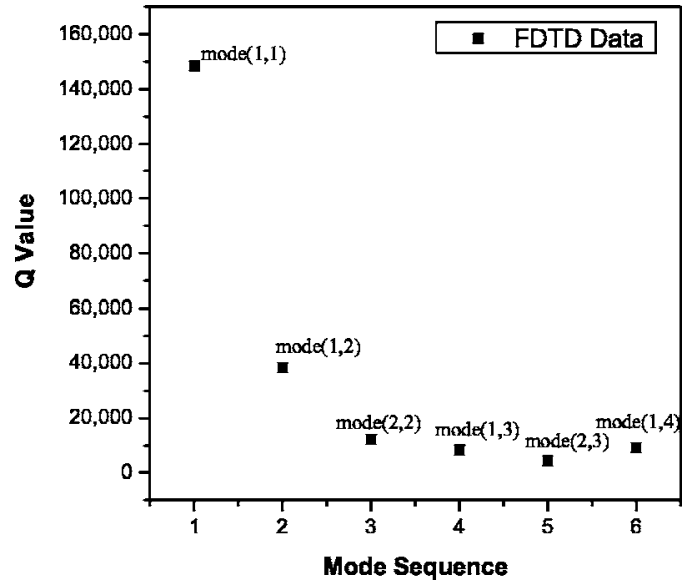

FIG. 10. The $Q$ values of the confined modes in a $13 \times 13 X$ structure.

near to the $M$ points in the first Brillouin zone and the spread of k becomes smaller; thus, the $Q$ values of the confined modes increase much faster than what might be anticipated from the 1-D Fabry-Perot model in $X$ structures. In conclusion, in $X$ structures, 1-D Fabry-Perot model breaks down and $\mathbf{k}$ anisotropy and TIR at the boundary account for the large $Q$ values that increase very fast with the size of the structure.

We obtained the $Q$ values of higher-order confined modes in a $13 \times 13 X$ structure and show them in Fig. 10. Away from the band edge, the confinement degrades because the spread of $\mathbf{k}$ is larger for higher-order modes. Since the $Q$ value of the fundamental mode, 148000 , is much larger than the second highest $Q$ value, 40000, in this frequency range, it is possible to achieve single-mode lasing operation using such a structure as a laser cavity.

\section{SUMMARY}

The optical properties of finite-size photonic crystals (PCs) are different from infinite ones. It is shown that, in finite-size structures, there exist isolated instead of a continuum of states. The "quantized" modes have the form of Bloch functions modulated by the envelope functions that vary on the length scale of the structure. We may describe the modes using the envelope function approximation (EFA) method analogous to the quantization of electron states in electronic crystals. EFA focuses on the modulating functions that encapsulate the general properties of underlying Bloch functions and thus is a physically intuitive and time-efficient treatment of finite-size PCs, especially for the 3-D case. Since there is no "attractive potential" in linear PCs, the coupling of light in PCs and in air at the boundary is important to the EFA method. In this paper, a vanishing boundary condition is assumed for the confined modes. The mode pattern and frequency position expected from EFA fit strikingly well with the ones from 2-D FDTD calculations in $X$ structures, while in $M$ structures, the discrepancy is larger. The results verify the applicability of EFA and reveal the importance of boundary conditions for further development of this method.
Lifetime is an important property of the confined modes in finite-size PCs, which cannot be directly calculated by the EFA method. We investigate the relationship of leakage rate, and the size and boundary orientation of the structure in finite-size PCs by FDTD. The $Q$ value of the fundamental mode around a band edge increases with the size of the structure due to the decrease in group velocity. Boundary orientation also influences the leakage rate. Because of $\mathbf{k}$ anisotropy at a band edge, we demonstrate that there is a total internal reflection (TIR) for certain boundary orientations, which is unique for 2-D and 3-D PCs. There is high confinement in such cases and we can consider the system "closed" for the confined modes in it, which justifies our assumption of a vanishing boundary condition and exposes the diversity of boundary conditions in 2-D and 3-D PCs for EFA development. We obtain $Q$ values of more than a million for the structures with only 19 rods along each boundary, which is about 190 times larger than that anticipated from group velocity analysis. Combining the group velocity decrease and TIR, we can design varieties of cavities, which are suitable for developing laser cavities and building structures with enhanced nonlinear optical interaction.

\section{ACKNOWLEDGMENTS}

We wish to acknowledge the helpful discussion about the FDTD calculation and band edge cavities with Dr. Alongkarn Chutinan in the Physics Department at the University of Toronto. We gratefully acknowledge financial support from CIPI, NSERC, CITO, MMO, and AFOSR.

\section{APPENDIX: MODIFICATION OF THE PARABOLIC DISPERSION RELATION AT THE BAND EDGE}

In this appendix we show how the parabolic dispersion relation at the first TM band edge, as illustrated in Fig. 2, can be modified by adding fourth-order terms to more accurately fit the real band surface further away from the band edge.

Normally it is assumed that near the band edge $\left(\mathbf{k}=\mathbf{k}_{M}\right)$, the dispersion relation is parabolic as expressed in the following equation:

$$
\omega_{\mathbf{k}}^{2}=\omega\left(\mathbf{k}_{M}\right)^{2}+\left.\sum_{\xi=x, y} \frac{\partial^{2} \omega^{2}}{\partial k_{\xi}^{2}}\right|_{\mathbf{k}_{M}}\left(k_{\xi}-k_{M \xi}\right)^{2} .
$$

In our case, this dispersion is isotropic because of crystal symmetry. However, in a real band structure, high-order terms are not always negligible and are required to account for the anisotropic properties of the dispersion surface at the band edge. In the cases of interest to us, away from the first TM band edge, the EFS becomes square instead of round as shown in Fig. 11. Along different directions, such as the $M$ $-\Gamma$ and $M-X$ directions, the dispersion curves are different. Thus the parabolic dispersion relation is inapplicable and one must include higher-order terms. From the symmetry of the lattice, it follows that the next-higher-order term is fourth order in $\mathbf{k}$ and is of the form 


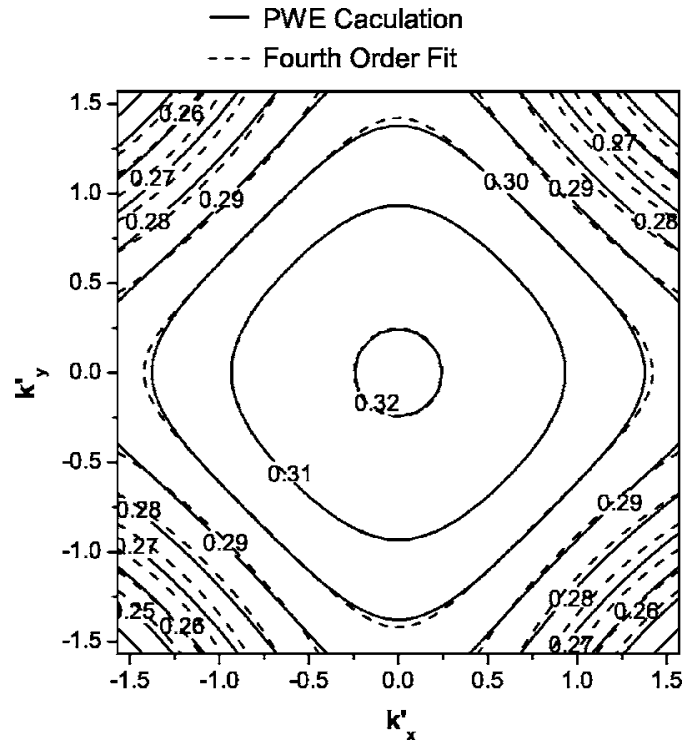

FIG. 11. Comparison between the EFS of the first TM band computed by the PWE method and that calculated from the fourthorder fit Eq. (A2). The fit parameters are the same as in Fig. 6. The unit of $\mathbf{k}$ is $1 / a$.

$$
\omega_{\mathbf{k}}^{2}=\omega_{1}\left(\mathbf{k}_{M}\right)^{2}+b\left(k_{x}^{\prime 2}+k_{y}^{\prime 2}\right)+c\left(k_{x}^{\prime 4}+k_{y}^{\prime 4}\right)+d k_{x}^{\prime 2} k_{y}^{\prime 2}
$$

where $k_{\xi}^{\prime}=k_{\xi}-k_{M \xi}$ and $x, y$ directions are the two orthogonal $M-X$ directions as shown in Fig. 11. The parameters $b, c$, and $d$ are defined as $b=\partial^{2} \omega_{1}^{2} / \partial k_{x}^{2}\left|\mathbf{k}_{M}, c=\partial^{4} \omega_{1}^{2} / \partial k_{x}^{4}\right| \mathbf{k}_{M}$, and $d$ $=\partial^{4} \omega_{1}^{2} /\left.\partial k_{x}^{2} \partial k_{y}^{2}\right|_{\mathbf{k}_{M}}$.

To show the agreement between the fourth-order fit and real EFS acquired from the PWE, we calculate the parameters in Eq. (A2) by fitting the dispersion curve along the $M-\Gamma$ and $M-X$ directions. Along the $M-\Gamma$ direction, the dispersion curve from fourth-order fit should be

$$
\omega_{\mathbf{k}}^{2}=\omega_{1}\left(\mathbf{k}_{M}\right)^{2}+b k_{M}^{\prime 2}+c / 2 k_{M}^{\prime 4}+d / 4 k_{M}^{\prime 4},
$$

where $k_{M}^{\prime}=\left|\mathbf{k}-\mathbf{k}_{M}\right|$.

Along the $M-X$ direction, the dispersion curve from fourth-order fit should be

$$
\omega_{\mathbf{k}}^{2}=\omega_{1}\left(\mathbf{k}_{M}\right)^{2}+b k_{X}^{\prime 2}+c k_{X}^{\prime 4} .
$$

By fitting the dispersion curves along these two directions, we may obtain the parameters $\omega_{1}\left(\mathbf{k}_{M}\right), b, c$, and $d$. In Fig. 11 the EFS from the PWE method and from the fourthorder fit are plotted together. As shown, the EFS from the fourth-order fit agrees well with the ones from the PWE for the frequency range from 0.28 to the band edge, which covers the frequency range of the confined modes in the finitesize PCs studied here.
${ }^{1}$ S. John, Phys. Rev. Lett. 58, 2486 (1987).

${ }^{2}$ E. Yablonovitch, Phys. Rev. Lett. 58, 2059 (1987).

${ }^{3}$ K. M. Leung and Y. F. Liu, Phys. Rev. Lett. 65, 2646 (1990).

${ }^{4}$ J. D. Joannopoulos, Photonic Crystals: Molding the Flow of Light (Princeton University Press, Princeton, NJ, 1995).

${ }^{5}$ K. Sakoda, Optical Properties of Photonic Crystals (Springer, Berlin, 2001).

${ }^{6}$ S. H. Kwon and Y. H. Lee, IEICE Trans. Electron. E87C, 308 (2004).

${ }^{7}$ M. Imada, S. Noda, A. Chutinan, T. Tokuda, M. Murata, and G. Sasaki, Appl. Phys. Lett. 75, 316 (1999).

${ }^{8}$ S. Noda, M. Yokoyama, M. Imada, A. Chutinan, and M. Mochizuki, Science 293, 1123 (2001).

${ }^{9}$ M. Imada, A. Chutinan, S. Noda, and M. Mochizuki, Phys. Rev. B 65, 195306 (2002).

${ }^{10}$ A. Yokoo, H. Suzuki, and M. Notomi, Jpn. J. Appl. Phys., Part 1 43, 4009 (2004)

${ }^{11}$ J. P. Dowling, M. Scalora, M. J. Bloemer, and C. M. Bowden, J. Appl. Phys. 75, 1896 (1994).

${ }^{12}$ K. Sakoda, K. Ohtaka, and T. Ueta, Opt. Express 4, 481 (1999).

${ }^{13}$ K. Sakoda, Opt. Express 4, 167 (1999).

${ }^{14}$ L. Florescu, K. Busch, and S. John, J. Opt. Soc. Am. B 19, 2215 (2002).

${ }^{15}$ K. Sakoda, J. Opt. Soc. Am. B 19, 2060 (2002).

${ }^{16}$ M. Soljacic, S. G. Johnson, S. Fan, M. Ibanescu, E. Ippen, and J. D. Joannopoulos, J. Opt. Soc. Am. B 19, 2052 (2002).
${ }^{17}$ M. Soljacic and J. D. Joannopoulos, Nat. Mater. 3, 211 (2004).

${ }^{18}$ G. Bastard, Wave Mechanics Applied to Semiconductor Heterostructures (Les Ulis, France; Editions de physique, France, 1988).

${ }^{19}$ M. G. Burt, J. Phys.: Condens. Matter 11, 53 (1999).

${ }^{20}$ S. G. Johnson and J. D. Joannopoulos, Opt. Express 8, 173 (2001).

${ }^{21}$ K. S. Yee, IEEE Trans. Antennas Propag. 14, 802 (1966).

${ }^{22}$ J. Vuckovic, M. Pelton, A. Scherer, and Y. Yamamoto, Phys. Rev. A 66, 023808 (2002).

${ }^{23}$ J. Vuckovic, M. Loncar, H. Mabuchi, and A. Schere, IEEE J. Quantum Electron. 38, 850 (2002).

${ }^{24}$ S. P. Guo and S. Albin, Opt. Express 11, 1080 (2003).

${ }^{25}$ K. Srinivasan and O. Painter, Opt. Express 10, 670 (2002).

${ }^{26}$ M. Boroditsky, R. Coccioli, and E. Yablonovitch, Proc. SPIE 3283, 184 (1998).

${ }^{27}$ A. Taflove and S. C. Hagness, Computational Electrodynamics: The Finite-Difference Time-Domain Method (Artech House, Norwood, MA, 2000).

${ }^{28}$ J. P. Berenger, J. Comput. Phys. 114, 185 (1994).

${ }^{29}$ M. R. Zunoubi, N. H. Younan, J. H. Beggs, and C. D. Taylor, IEEE Trans. Electromagn. Compat. 39, 247 (1997).

${ }^{30}$ M. D. Feit and J. A. Fleck, Appl. Opt. 19, 1154 (1980).

${ }^{31}$ M. Charbonneau-Lefort, E. Istrate, M. Allard, J. Poon, and E. H. Sargent, Phys. Rev. B 65, 125318 (2002).

${ }^{32}$ E. Istrate, M. Charbonneau-Lefort, and E. H. Sargent, Phys. Rev. 
B 66, 075121 (2002).

${ }^{33}$ J. Poon, E. Istrate, M. Allard, and E. H. Sargent, IEEE J. Quantum Electron. 39, 778 (2003).

${ }^{34}$ M. Notomi, Phys. Rev. B 62, 10696 (2000).

${ }^{35}$ M. G. Burt, J. Phys.: Condens. Matter 4, 6651 (1992).
${ }^{36}$ B. A. Foreman, Phys. Rev. Lett. 80, 3823 (1998).

${ }^{37}$ S. V. Nair, L. M. Ramaniah, and K. C. Rustagi, Phys. Rev. B 45, 5969 (1992).

${ }^{38}$ A. Yariv, Optical Electronics in Modern Communications (Oxford, New York, 1997). 